\title{
The Importance of Treating Energy Imbalances and Chakras Replenishment for Prevention and Treatment of Cancer
}

\author{
Huang WL* \\ Medical Acupuncture and Pain Management Clinic, Franca, São Paulo, Brazil.
}

*Corresponding author: Huang WL, Medical Acupuncture and Pain Management Clinic,

Received Date: August 19, 2020

Franca, São Paulo, Brazil.

\begin{abstract}
Introduction: Western medicine's cancer treatment focus on the destruction of the diseased cells, harming the healthy cells as well. In traditional Chinese medicine (TCM), cancer is the manifestation of the body's energy deficiency and Heat retention. One direct way to search for the root of these energy imbalances is through the assessment of the chakra system, which offers vital information about the body's energy levels.

Purpose: To demonstrate that cancer patients have energy deficiencies and Heat retention as root and also chakra's energy deficiencies in the lowest level. The balance of the energy deficiencies and taking out the Heat retention associated with the replenishment of these energies is important to prevent and treat cancer patients.

Methods: Three clinical cases reports. All three patients with cancer diagnoses (case one: thyroid; case two: uterus; case three: lungs). All of them received treatment to reestablish the equilibrium between Yin, Yang, Qi, Blood and taking out Heat retention through Chinese dietary counselling, auricular acupuncture with apex-ear bloodletting, homeopathy according to the Constitutional Homeopathy of Five Elements Based on Traditional Chinese Medicine and crystal-based medication after chakras energy measurement through radiesthesia procedure. All three patients were found to be at their lowest level of energy, rating one out of eight. Results: Out of the three patients, the first two case reports were cured of their cancer condition without any treatment by Western medicine, only with the treatment done. The third patient, though, was already under radiotherapy and chemotherapy but through the treatment previously described the metastasis disappeared and he achieved a better physical and emotional health state.
\end{abstract}

Conclusion: TCM believes that energy deficiency and Heat retention causes cancer. Treatment with Chinese dietary counseling, auricular acupuncture, apex-ear bloodletting and recharge of the Chakras energy can be a path to prevention and treatment of cancer patients.

Keywords: Energy; Chakras; Five Elements Theory; Traditional Chinese Medicine; Chinese nutritional therapy; Acupuncture; Apex-ear bloodletting; Moxibustion; Hippocrates; Cancer

\section{Introduction}

Cancer is an abnormal propagation of cells in the body, counting up to about one hundred different types and manifestations of the disease. [1,2] While the focus of Western medicine is on the pathology itself $[1,2]$, the physician's primal concern is what kind of tumor the patient has, benign or malignant. [1,2] In the article "TCM and cancer: History, present situation, and development" by Jie Liu, Shuo Wang,Ying Zhang, Hui-ting Fan, and Hong-sheng Lin, it is stated that for cancer patients in an advanced stage or elderly patients, for any of those whom standard treatment is unsuitable, TCM comes to stabilizes tumor injuries, improve the patient's overall wellbeing, enhancing the patient's quality of life in a prolonged survival time. The authors also cite that while a 
physician is looking for a preventive treatment before the disease is set, if TCM treatment is applied in an early stage, it can heal precancerous lesions and reduce the incidence of cancer itself. [3] For the construction of the reasoning the author took to treat all her patients and, in this case,, specifically, cancer patients, the author based her treatment in a specific case of a patient the author had in 2006. This patient was a 70-year-old patient, who reported pain in the legs and was diagnosed with Kidney-Yang deficiency, according to TCM. He received treatment with Chinese dietary counseling, acupuncture and auricular acupuncture associated with apex ear bloodletting. [4-7] With the TCM treatment applied, the patient reported back that the pain in the legs diminished. In his follow-up consult, 30 days after, the patient revealed that his eye pressure had also lowered with the treatment, as his ophthalmologist confirmed. During the treatment, he had not reported to be treating glaucoma in the last 40 years with no improvement of his condition. With the treatment performed, his eye pressure lowers from $40 \mathrm{mmHg}$ to 17mmHg. [4-7] This interesting case became the cornerstone of the author studies in the field, trying to comprehend how the treatment based on the root of the problem could treat different diseases and symptoms simultaneously and using the same methods. [4-7] In this study the author is trying to demonstrate that the treatment of the energy imbalances in the root level can treat patients with cancer, achieving a cure or control the formation of metastasis as well giving to the patient better quality of life regarding their physical and emotional aspects.

\section{Purpose}

The purpose of this study is to demonstrate that cancer patients have energy deficiencies with Heat retention as the root of the problem associated with an important chakras energy meridian deficiencies in common and the correction of these energy imbalances and taking out the Heat retention, associated with the replenishment of the Chakras' energy meridians is an important tool in the prevention of patients without cancer and treatment of cancer patients, associated or not with Western medicine approaches.

\section{Methods and Results}

Literature regarding cancer causes and treatment on Western medicine and traditional Chinese medicine were also analyzed. All analyzed articles were PubMed indexed. Three case reports are also presented in this article.

\section{Case Report One}

In November 2019, the patient named N.M.J.G, a 70-year-old woman did a routine gynecological exam, which led the doctor to ask for a thyroid ultrasound exam. On the results, it was found that the patient had three tumors on the thyroid-the biggest with 14 centimeters. The patient was submitted to a biopsy which confirmed the diagnosis of malignance of the tumors. She was taken to a head and neck specialist medical doctor, which requested an urgent surgery to take off all the thyroid and begin the iodo therapy and chemotherapy afterwards. She started treatment with Levothyroxin, prescribed by him. Stressed out by her recent diagnoses, the patient sought the author's clinic after three years without acupuncture treatment she used to do. The patient also had diabetes, obesity, hypertension and diabetic retinopathy. Once she came back to the treatment her TCM diagnoses was Yin deficiency and Heat retention. Based on TCM reasoning, the author oriented her to take out of her diet all dairy products, avoid cold liquids, raw foods and sweets. It was also advised to avoid fried foods, chocolate, eggs, coconut, honey, pepper, alcoholic beverages. The last group of drinks that she needed to avoid was coffee, matte tea and soda. Her treatment also included auricular acupuncture daily with apex ear bloodletting procedure. She was also submitted to the radiesthesia procedure through a crystal pendulum to analyze the level of energy of her chakras energy meridian. All her seven chakras appeared very depleted, rating one out of eight. The patient was prescribed homeopathy based on the author's theory "Constitutional Homeopathy of the Five Elements based on Traditional Chinese Medicine" and crystal based medications for a period of at least one year; after this period, another chakras energy measurement would be done to see how was her chakras' energy improvement.

The medication used will be described in the discussion session.

\section{Results}

After three months of treatment, the size of the tumor was dramatically reduced, and the patient noticed that her neck became to look normal again (as the tumor was visible externally in the beginning). The ultrasound exams showed reduction of the tumor, from 14 centimeters to 4.5 centimeters, and from the other two tumors, one disappeared. Even with the reduction in the size of the tumor, the patient was indicated, as scheduled from the beginning, to undergo surgery to remove the thyroid gland. During the surgery, it was once again confirmed that one out of the three tumors had disappeared. A biopsy was performed by freezing and to the surgeon's surprise, the diagnose of the tumor, previously diagnosed as malignant, had become benign. After this discovery, the thyroid of the patient that needed to be completed removed at first moment, was now normally functioning, not needing to be taken out surgically. The patient did not need to do chemotherapy or iodotherapy anymore. In addition to this improvement, the patient also completely improved the condition of diabetic retinopathy in her eye; as well as an improvement in the glycemic control of the patient's diabetes that used to be high $(>250 \mathrm{mg} /$ dl). Currently, the patient's blood glucose is around $110 \mathrm{mg} / \mathrm{dl}$ and hemoglobin glycosylated (which used to be, on average, 9.2\% is currently in 6\%); the endocrinologist accompanying the patient wishes to remove the insulin that the patient applies, due to the great improvement in her case. 


\section{Case Report Two}

A 38-year-old female, half Japanese half Brazilian, a podiatrist, came to the author's clinic after having a pap smear exam showing atypical cellular results. The patient was frightened by the idea of cancer. Her mother was already a patient of the author's and suggested a consultation. At her first consultation, her diagnosis was, Yin, Yang and Qi and Blood Deficiency and Heat retention according to TCM. Her treatment consisted in: Chinese dietary counseling, avoiding all dairy products, cold liquids, raw foods and sweets. It was also advised to avoid fried foods, chocolate, eggs, coconut, honey, alcoholic beverages. Lastly, the drinks to be avoided were coffee, matte tea and soda. She also received acupuncture and auricular acupuncture with apex-ear bloodletting procedure twice a week. The patient's chakras were measured through the radiesthesia procedure using a crystal pendulum and they were all rating one out of eight, with the exception of her seventh chakra, that was normal at rate. Homeopathy according to Constitutional Homeopathy of Five Elements Based on Traditional Chinese Medicine and crystal-based medications for the replenishment of the energy of the chakras were also prescribed for a period of at least one year where after this period another chakras' energy measurement was prescribed to be done.

\section{Results}

Within less than eight acupuncture sessions, the patient was able to normalize her exams, and the atypical cells disappeared on her new pap smear exam. Even after the normalization of her exam, the author suggested that she continued her treatment with auricular acupuncture with apex-ear bloodletting, maintaining all her diet therapy and all homeopathy and crystal-based medications prescribed to prevent the recurrence of the tumor or the appearance of new tumors on another sites.

\section{Case Report Three}

Patient R.A.B, 42-year-old male, a veterinarian doctor, first complained of breathlessness, back pain and consistent cough in March 2015. The first physician to be sought was a pulmonologist, where besides pneumonia being confirmed, a white spot on the right lung was identified on an x-ray. The patient was then first admitted for the protocol examination, for the physician thought the white spot could be a tumor. A month later, pneumonia symptoms returned, this time accompanied by severe chest pains (pricking). A bronchoscopy was performed, with no results. The patient then sought an oncologist at the Sírio Libanês hospital in São Paulo, $400 \mathrm{~km}$ from the city where he lived. The new doctor asked for a review of all tests performed until that moment. A biopsy in the mediastinum region was performed, next to the pleura of the right lung, where it was found dead cells from the mediastinum region to be removed surgically. In Western medicine, his diagnosis was small cell neuroendocrine carcinoma. The patient had his first chemotherapy session with Cisplatin and Etoposide, at intervals of 21 days for 6 months, until December 2015. After 3 months of recovery, PET SCAN and CT were requested, then verifying the decrease in tumor size. Four months later, in July 2016, new tests were performed and metastasis was confirmed in the pancreas and stomach. The second round of chemotherapy was recommended, with the medication Carboplatin and Etoposide intravenously, in regular sessions with intervals of 21 days for 6 months, until January 2017. New tests were performed and the reduction of tumors were again confirmed. Unfortunately, three months later, the patient began to experience new "weird symptoms", such as difficulty speaking. An urgent MRI scan had been requested, where a new tumor was found, in the brain, with a few small lumps scattered. The patient was promptly referred for neurosurgery, at the 9 de Julho hospital in São Paulo, at the end of May 2017. After the surgery, radiotherapy in the head was indicated for 30 days. One day, the patient was experiencing severe dyspnea and at the request of his mother, he went to the clinic of the author of this study for evaluation. The patient was afraid that shortness of breath could be a recurrence of his lung tumor, but after the author's assessment, a session of auricular acupuncture with apex-ear bloodletting and moxibustion were done. A second assessment using radiesthesia was performed by the author to assess the energy of the patient's chakras. The result confirmed what the author predicted that all the patient's chakras were without energy. Then, a nutritional therapy was prescribed, in which the patient was to avoid all dairy products, cold water, raw foods, sweets, coffee, matte tea, soda, in addition to reducing other foods that could cause damage to the liver's energy such as fried food, eggs, chocolate, coconut and alcoholic beverages. The patient had confessed to the doctor that he was very fond of foods with melted cheese, such as sandwiches from Mc Donald's and pizzas, therefore, the author advised to avoid any and all foods that contained melted cheese. Auricular acupuncture with apex-ear bloodletting and moxibustion were prescribed by the author twice a week, and homeopathy according to this author's "Constitutional Homeopathy of the Five Elements based on Traditional Chinese Medicine", crystal-based medications, all with the goal to replenish the weakened chakras Energy.

\section{Result of Case Three}

After the first acupuncture session, his appearance was much better, the dyspnea had improved a lot, making the patient less anxious, as the patient thought his shortness of breath could be related to his tumor growing again, however, the author had explained to the patient that the shortness of breath he was experiencing was related to the lack of energy in the chakras. The patient's improvement was gradual and increasing with each session with the author, taking the highly diluted medications (homeopathy and crystal-based medication), becoming stronger, noting the gradual reduction of some tumors that he had in the 
cervical region and on the anterior abdominal wall, even noticing the total disappearance of them. Currently, the patient continues to use his chemotherapy medications, prescribed by his oncologists, but performing with the author, in parallel, the energy replacement treatment of the chakras, seeking to improve his vital energy that was weakened. The author, in several infectious or non-infectious symptoms (gastroesophageal reflux, loss of appetite, anxiety, insomnia, etc.), always resorted to the use of Chinese dietary therapy, guiding the patient to the correct time for the correct feeding according to the symptoms presented. In addition, she has always used acupuncture to rebalance the patient's energy to avoid giving other medications (all kinds of high concentrated medications) that could cause a reduction in vital energy, which may lead to tumor recurrence or new formation in another location. The same reasoning is due to the use of highly diluted medication (homeopathy and crystal-based medication) in order to always improve vital energy and in this way strength the energy itself that could fight the tumor and prevent the formation of new tumors in the future. After two years of treatment replenishing his energy using TCM tools and high diluted medication, the patient was happy to be healthy enough to have his girlfriend pregnant.

\section{Discussion}

Amid 2010 and 2020, the statistics of confirmed cancer diagnoses in the USA are expected to go up about $24 \%$ in men, summarizing to more than 1 million cases per year. With women, the rates are expected to rise $21 \%$ to 900,000 new cases per year. [8] As already stated, cancer is categorized by unrestrained cell growth and attainment of metastatic features. Generally, instigation of oncogenes and/or deactivation of tumor oppressor genetic factors lead to unrestrained cell cycle advancement and inactivation of apoptotic mechanisms. [8,9] Malignant tumors, therefore, cancers, acquire metastasis, which happens partially due to the downregulation of cell connection receptors essential for tissue specific and cell to cell attachment. In addition, stimulation of membrane metalloproteases offers a pathway for the spreading of metastatic cells, propagating the cancer disease. [8,9] What is already known as a trigger for the cancer cells to develop and spread, it's tobacco. Other statistics point out cancer deaths can also be linked to obesity, poor nutrition, lack of physical activities and substantial alcohol consumption; Cancer can also be genetic, especially in the breasts between women of the same family. $[8,9]$ In Western medicine, cancer is often treated with radiation, chemotherapy, targeted therapy and surgery. [8,9] Normally, on a daily basis, our body produces malignant cells. But when our immune system is intact, it takes care of eliminating these cells from our body. In this study, the author demonstrates that patients who have cancer, have an energy deficiency, and how energy is directly related to immunology, as shown in several studies, it is very likely that there will be a formation and growth of cancer in these patients due to this energy weakness, which lead to a weakness of the immune system itself at first. [10] In the last 50 years, scientific studies summarizing clinical practice has established that the use of TCM in cancer therapy has reduced toxicity and heightened the efficacy of radio chemotherapy, sustaining survival in cancer patients in advanced phases, as well as aided in the prevention and treatment of reappearances and metastasis. [8,9] Our predecessor's acknowledged that cancer etiology involved exopathogens, environment, emotional issues and inadequate diet: "with discomfort, improper diet, cold temperature from time to time, pathogens prevail and accumulation has left" (The Yellow Emperor's Inner Canon, English translation). [11] TCM strategies for cancer are a combination of partial and systemic therapy, seeking the strengthening of the body's resistance while eliminating pathogens, treating the body and emotion at the same time, Heat clearance and detoxification, activation and removal of Blood stasis. Radiotherapy and chemotherapy can damage $Q i$ and Blood, it's core and fluids and the function of internal organs. [8,9] TCM can improve the clinical efficacy of radiotherapy and chemotherapy, guided by therapeutic principles of tonifying $Q i$ and producing Blood, strengthening the Spleen and Stomach, nourishing the Liver and Kidney eliminating Heat from Blood and toxic material from the body. TCM associated with chemotherapy has revealed promising results in improving efficacy with less side effects prolonging the survival time of postoperative cancer patients. [8,9]

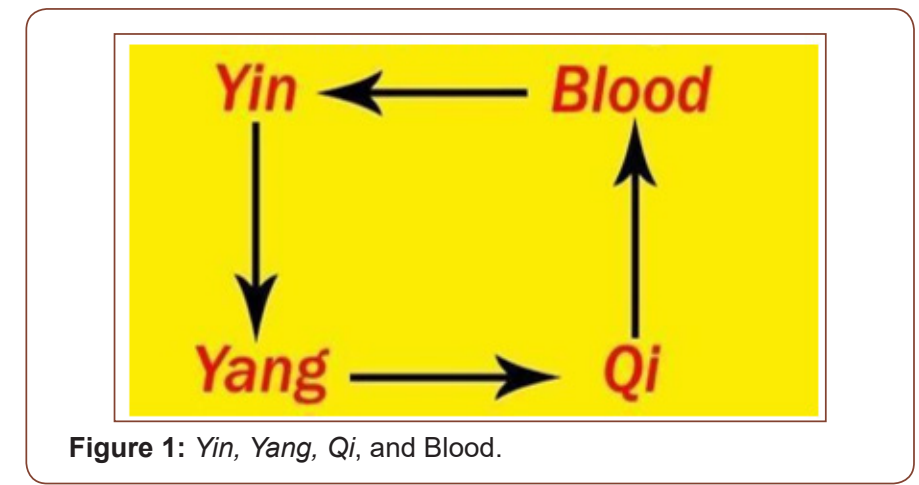

TCM applied at an initial stage can treat precancerous lesions and lessen the incidence of cancer, that said, on treatment applied before the disease is set. Cancer in the view of traditional Chinese medicine is considered a possibly curable disease as well as a preventable. [12] In order for us to understand cancer; the author assume that all formation of "masses" in our body is considered Phlegm. Phlegm is the product of a dysfunction of the Spleenpancreas. When the Spleen-pancreas becomes weakened for some reason, the internal humidity that remains trapped in our body, over time, ceases to be fluid, becoming a viscous mass that TCM call Phlegm. [12] From the perspective of TCM, cancer is caused by energy deficiency and Heat retention [13] and a tumor is the uppermost branch of the illness, but not the root. Each cancer patient may have different energy imbalances causing the same 
type of cancer. [13] In this article, the author is showing in the three case reports that what the three cases have in common is the profound lack of energy in all the chakras energy meridians, leading to a weak immune system that is normally responsible for the clearance of the malignant cells produced every day. There are four main energies that are responsible for health: Yin, Yang, Qi and Blood [14,18] (Figure 1).

Any imbalance between these four energies can lead to a fifth imbalance, which is the formation of internal Heat. $[14,18]$ The patient who has internal Heat manifested clinically as bad breath, bleeding gums, red skin lesions, acne like, abdominal pain, microscopic hematuria, may be an indirect indication of clinical predisposition for cancer development in the future - or the patient may already have it, as this may be a clinical indication that the patient is deficient in energy, which in TCM, together with Heat retention, is the cause of cancer formation. $[14,18]$ So, in addition to all the guidelines made by Western medicine in terms of correcting the diet and readjusting the lifestyle to avoid or improve the treatment of cancer patients, from an energy point of view, the author usually avoid all situations that lead to energy deficiencies or increase Heat retention. $[14,18]$ The author explains that from health to disease, there are five phases the patient goes through. During phases 1 to 3 , there is a slowing of the organ functions due to energy imbalances, and the patient has and complains of symptoms, but the results of laboratorial exams are normal (Table 1). At phase 4 the laboratory exams show some alterations, and the disease is still curable, and at phase 5 , the exams are very altered, and the cellular damage is irreversible and the disease incurable (Table 1).

In this study, the author demonstrates through case reports that the development of the disease phases from 1 to 5 can occur in one direction but also regress from phase 5 to 4 or from phase 4 to 3 , as occurred with the case patient report 1 , who had a malignant cancer and after the treatment performed, her diagnosis became benign; that is, the patient was in phase 5 (incurable) and moved to phase 4 (curable). Although spontaneous cancer regression is defined as remission or disappearance of a tumor in the absence of any therapeutic intervention, today it is believed that this is caused by immunologically important events. In the article entitled "Spontaneous tumor regression" written by Tarik Salman and published by the Journal of Oncological Science, in April 2016, it states that spontaneous cancer regression could be caused by immunologically important events. [15] In the case report one, the patient with malignant thyroid cancer didn't received her chemotherapy or radiotherapy or surgery prior to her remission. The only treatment she received was the re-balancing of her internal energies using Chinese dietary counseling, as described in the case report, auricular acupuncture associated with apex ear bloodletting and systemic acupuncture. The second step in her treatment was to replenish the chakras energy that were completely empty. This kind of treatment achieved a very good result in this patient because in TCM, cancer can grow when there is low energy and formation of internal Heat, and this patient had all these two criteria (low energy and internal Heat). When the author treats this condition that was favorable for cancer growing in the beginning and took out all the factors creating another environment that disfavor the cancer growing, and in other words, improving the immune system as the energy is directly related to immune system, as found in other articles in the literature.

The case report 2 patient also had an exam showing that she was in phase 5 and after treatment, the patient did not show any other laboratory abnormalities: therefore, regressing from phase 5 to phase 3 . The case report 3 patient was in phase 5 and continues in phase 5, but with a much higher quality of life and reduced metastasis with the disappearance of many tumors. When the patient was undergoing treatment with chemotherapy and radiotherapy alone, there was a reduction in metastases but some reappeared in other parts of the body, this no longer occurred when he combined TCM's treatment to restore the energy of the chakras'. The method and its success is due to treating the patient holistically, at the root of the problem; taking into consideration all that is known nowadays in the treatment of cancer in Western medicine and TCM. The treatment of energy deficiency in cancer patients provides an improvement in the quality of life and accelerates the healing process, helping to reduce the size of the tumor and metastasis, as presented in the case report three patient. $[9,12,14,18]$ In this study, the author emphasizes the idea of treating the patient as a whole in the energy point of view, because of the root of the energy problems continues unbalanced and deficient in patients undergoing cancer treatment with chemotherapy, radiotherapy or surgery, and treatment replenishing these energies and taking out Heat retention is important to prevent the formation of a new cancer or metastasis, or helping the treatment of cancer itself; as the local and systemic treatment leads to a faster improvement of the process, often providing a reduction on tumor size (case report three) or a cure (case report one and two). To create a parallel on how diseases are diagnosed and treated today from the point of view of Western medicine and according to TCM, the author created the tree metaphor. In other articles, the author stated the importance of this metaphor, to address how Western and ancient perspectives could work together. TCM explains the functioning of the human body through a holistic view. Therefore, external factors such as Wind, Cold, Heat, Dryness, Humidity, diet and internal factors such as emotions may have an influence in the wellbeing. $[9,12,14,18]$ The tree has a trunk with several branches. Each branch represents each medical specialty and each leaf that comes out of each branch represents the symptoms and diseases of each specialty. In the case of case, Western medicine is treating at the leaf level (Figure 2). 


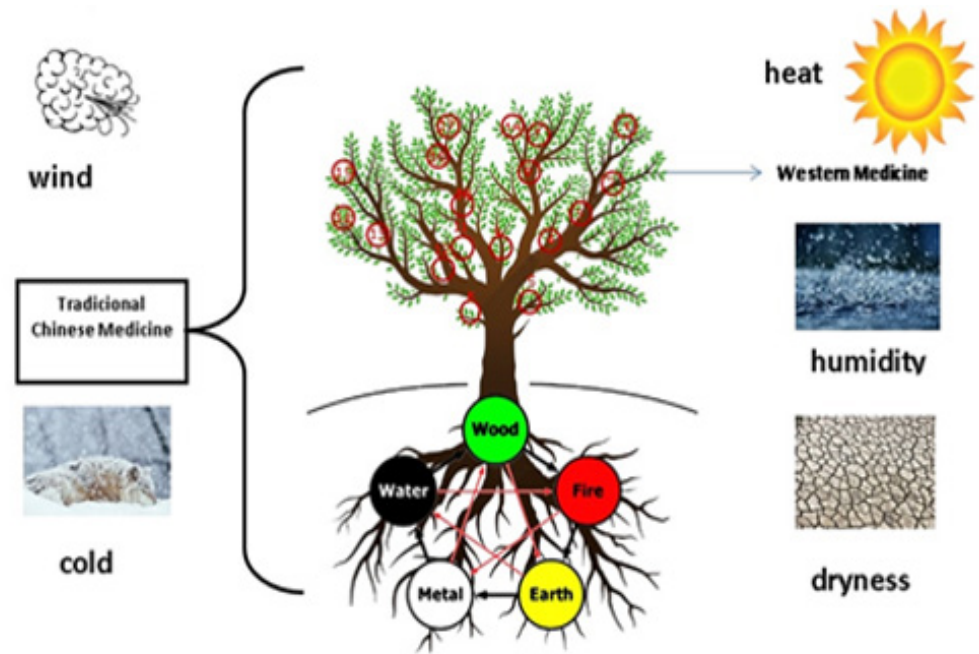

Figure 2: Schematic Relationship between TCM and Western medicine.

In the case of cancer, it is on the branch of oncology and the cancer is considered a leaf of the tree. The part of the tree that is below the earth - invisible to the naked eye - are the roots of the tree. This part remains unknown to Western medicine mainly because its existence is not recognized or discussed in the medical faculties. The roots of the tree represent the theory of Yin and Yang and Five Elements Theory. To be healthy, TCM aim for a state of equilibrium between the Yin and Yang energies. If there are imbalances between these two energies, the appearance of symptoms occurs, and if not properly treated, consolidates the disease in the future, in this study, for example, the disease could be a cancer. Everything in the universe is composed by Yin and Yang (Figure 3), including the human body. The balanced arrangement of the dark Yin and the light Yang is not static. The two points indicate the notion that when a force reaches its extreme point, it reveals within itself the seed of its opposite, their dynamic interaction is the essence of all-natural phenomena and all human situations. Opposites are complementary (Figure 3,4).

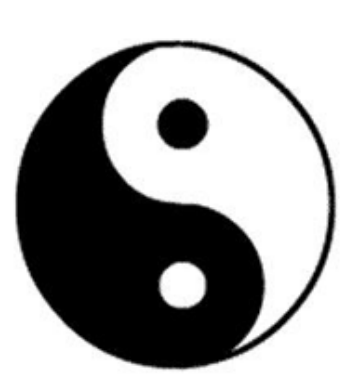

Figure 3: Yin and Yang Symbol.

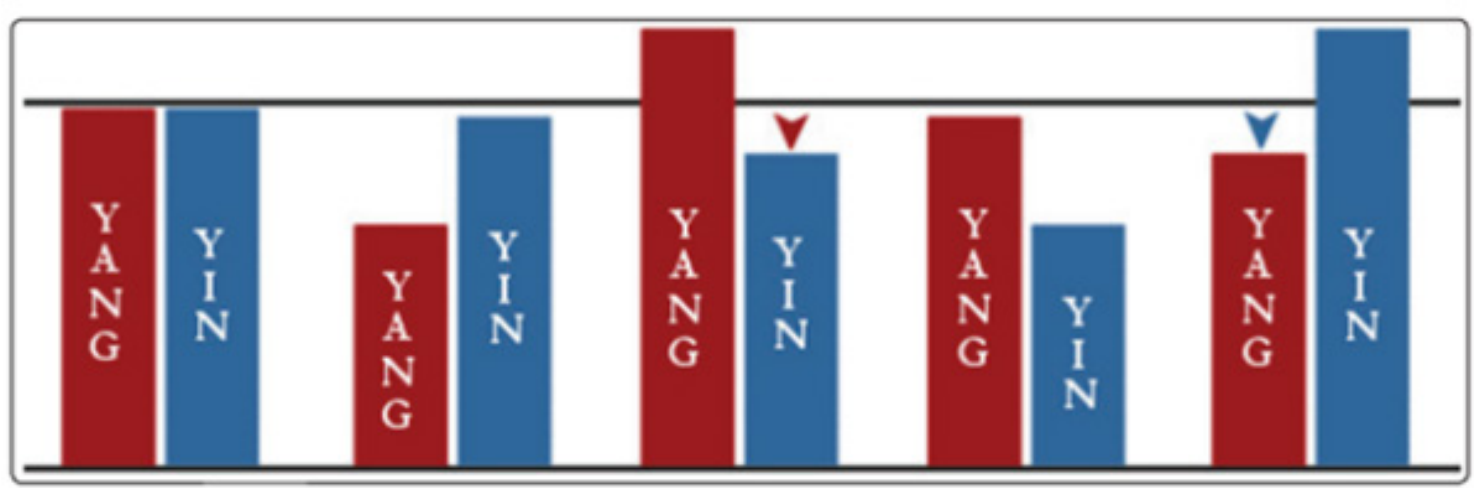

Figure 4: Balance and Disharmony between Yin and Yang. 
It is impossible to understand TCM without comprehending the main concepts of Yin and Yang. These concepts are the basis of all TCM reasoning. When Yin is dominant, it forces Yang into weakness. A dominance of Yang forces Yin into a state of weakness. If there is a weakness of Yin, Yang appears dominant; similarly, if Yang is weak, then Yin will appear stronger, as showed in figure 4. Inside the body, Yin is associated with organ tissue, while Yang is associated with organ function. With a Yin-deficiency, organs are deficient in nourishment, while a Yang-deficiency reflects a functional or performance deficiency in an organ or system. [18] The intention of all types of treatment according to TCM is to balance the Yin and Yang energy represented by the first red and blue column on the left side of Figure 4, where Yin and Yang must be at the same height, that is, the two energies in balance. In this way, the author was successful in treating the patients described in this article only by balancing these energies through the various types of treatment (Chinese nutritional therapy, auricular acupuncture with apexear bloodletting, moxibustion, homeopathy and crystal-based medication). [18] In addition to the Yin and Yang energy there is also the Blood and Qi energy so that Yin and Yang can flow through the body. [18] For Blood to free flow inside the vessel, another energy force must work in harmony with it, this energy is $Q i$. $Q i$ is the vital energy that invigorates Blood to keep it circulating throughout the body. Blood and Qi have a very important relationship and are mutually dependent, Qi prevents Blood stagnation, and Blood nourishes Qi [18] (Figure 5).

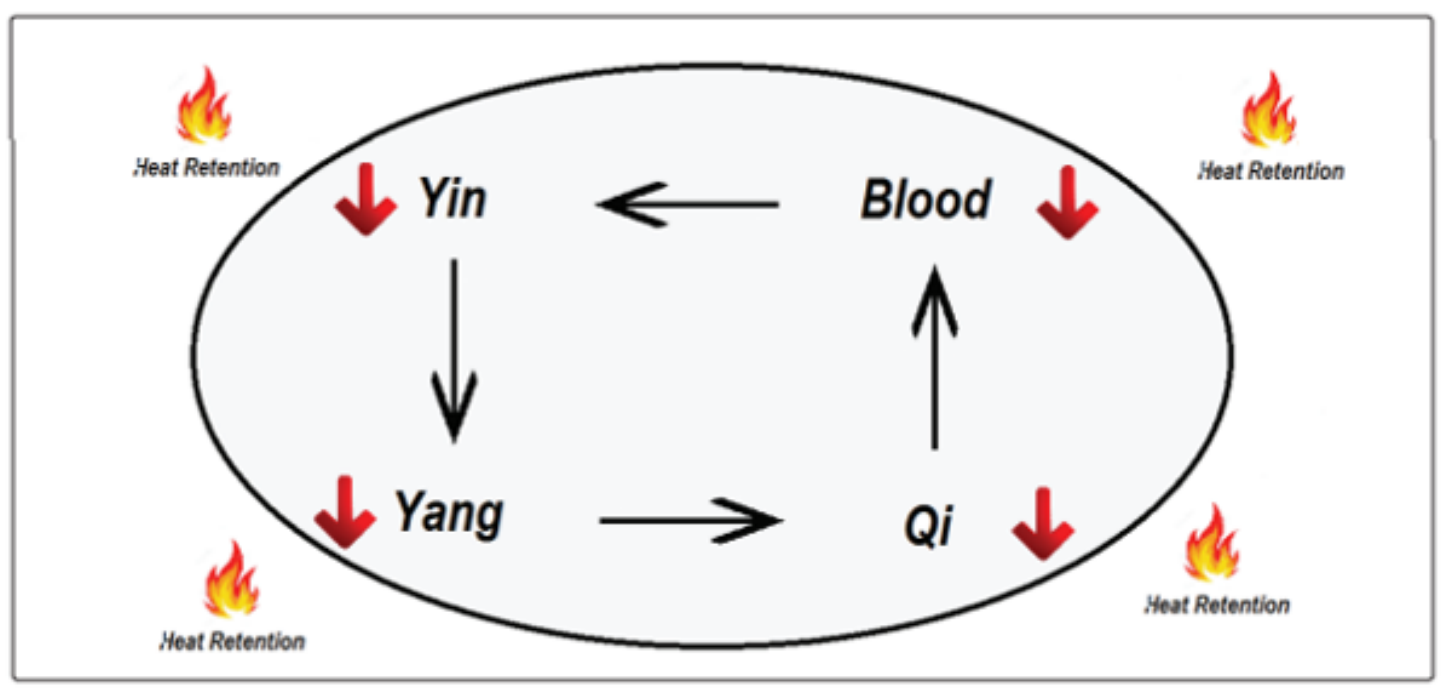

Figure 5: Internal Heat Formation in the Yin, Yang, Qi and Blood relation.

Internal Heat is an energy imbalance, generated by deficiency of one of Yin, Yang, Qi and Blood energies or a combination of imbalances of these energies. It can also be caused by incorrect diet, such as the constant consumption of fried foods, melted cheese, eggs, chocolate, coconut, honey and alcoholic beverages. Internal Heat can also be caused by excessive anger that leads to an increase of the Liver-Yang. A deficiency in one of these four elements, for example, a Yin deficiency, as you can see in figure 5, will generate other imbalances in the other three factors. Any imbalance in any of the four energies can generate internal Heat, that is the cause of cancer according to TCM. [18] Many times, only taking out the Heat retention and tone the energies that are deficient is sufficient to treat the cellular alterations presented in cancer patients. $[9,18]$ The other theory based upon TCM principles of treatment is the five-element theory. The Chinese wise men were able to observe the relation between the five basic elements: Wood, Fire, Earth, Metal and Water and a dynamic balance and relationship among these elements. [18] The theory of the 5 elements and how the interaction of the elements works has already been extensively explained by the author in several of her published articles. Quoting some, "Why Are Diabetic Patients Still Having Hyperglycemia despite Diet Regulation, Antiglycemic Medication and Insulin? ", Published in the International Journal of Diabetes \& Metabolic Disorders in March 2019;" Can We Treat Atopic Dermatitis without using Corticosteroids? "Published in the Journal of Pediatrics and Infants Volume 1: 2 of 2019; among many others - the author explains how the interaction of the five elements represented by the five massive organs (Liver, Heart, Spleen, Lung, Kidney) occurs within the body, demonstrating that the functioning of all organs is interrelated differently from the reasoning of Western medicine, where this view is different, the organs works separately and independently. [18] Every patient who undergoes treatment with the author, she makes the diagnosis by Western medicine (leaf level) and the diagnosis in Chinese medicine (root level). The diagnosis in Chinese medicine is based on the questioning of four phrases to know whether or not there are changes in the Yin, Yang, Qi, Blood and Heat retention energies, as shown in Table 2 (Table 2). 
Table 1: Progression of Health to Disease.

\begin{tabular}{|c|c|c|c|c|}
\hline \multicolumn{2}{|c|}{ Organ } & Exams & Energy Reserve & Symptom \\
\hline Phase 1 & Slowing down of organ functions & Normal & Energy Reserves-normal & Without clinical symptoms \\
\hline Phase 2 & Slowing down of organ functions & Normal & $\begin{array}{c}\text { Consumption of internal Energy } \\
\text { Reserves }\end{array}$ & With symptoms in other organ \\
\hline Phase 3 & Slowing down of organ functions & Normal & $\begin{array}{c}\text { Consumption of external Energy } \\
\text { Reserves }\end{array}$ & With symptoms in same organ \\
\hline Phase 4 & Reversible cellular lesion & Little alteration & Consumption of blood Reserves & Curable disease \\
\hline Phase 5 & Irreversible cellular lesion & Excessive alteration & Metabolic exhaustion & Incurable disease \\
\hline
\end{tabular}

Table 2: How to Diagnose Yin, Yang, Qi, Blood and Heat Retention Imbalances.

\begin{tabular}{|c|c|c|c|c|c|}
\hline Question & $\begin{array}{c}\text { Does the patient have a } \\
\text { daily bowel movement? }\end{array}$ & $\begin{array}{c}\text { Does the patient } \\
\text { have excessive } \\
\text { sweating during } \\
\text { day? }\end{array}$ & $\begin{array}{c}\text { Does the patient feel } \\
\text { cold in the extrem- } \\
\text { ities of the body? } \\
\text { (Cold feet or hands) }\end{array}$ & $\begin{array}{c}\text { Does the patient } \\
\text { feel hot in the } \\
\text { extremities? }\end{array}$ & $\begin{array}{c}\text { Does the patient has dry mouth, bleeding } \\
\text { gums, bad breath, acne and/or redness in } \\
\text { the skin, abdominal pain, micro hematuria, } \\
\text { or itching? }\end{array}$ \\
\hline Meaning & $\begin{array}{c}\text { The lack of daily bowel } \\
\text { movements can mean } \\
\text { Blood deficiency. }\end{array}$ & $\begin{array}{c}\text { This could be a } \\
\text { symptom of } Q i \\
\text { deficiency. }\end{array}$ & $\begin{array}{c}\text { Commonly, this is } \\
\text { a sign of Yang defi- } \\
\text { ciency. }\end{array}$ & $\begin{array}{c}\text { Commonly a sign } \\
\text { of Yin deficiency. }\end{array}$ & Commonly this is a sign of Heat retention. \\
\hline
\end{tabular}

Following the guidelines of Hippocrates, father of medicine, who says we should "Let food be thy medicine and medicine be thy food", the author uses diet as one of the main forms of treatment to maintain energy balance [19]. Based on Chinese nutritional therapy, all three patients from the previous case reports were oriented to take out of their diet all dairy products, avoid cold liquids, raw foods and sweets, to treat the imbalance of energy in the Spleen-pancreas, which is responsible for absorbing nutrients and forming Blood to nourish all organs, cells and tissues. If this meridian is unbalanced, nutrient absorption will not occur, and thus, it will be more difficult to promote the energy filling of the five massive organs (Liver, Heart, Spleen, Lungs and Kidney) that are without energy, as diagnosed by radiesthesia. Therefore, in order to rebalance this meridian, it is recommended to drink warm water, eat more cooked and sautéed foods and remove all dairy products and sweets. The imbalance of this Spleen-pancreas meridian is responsible for the formation of Phlegm, which is responsible for the formation of a tumor, according to TCM. [20] Therefore, by rebalancing this meridian, Phlegm, formation does not occur, and the tumors decrease in size until they disappear, as happened in the three clinical case report. Patients were also instructed to avoid drinking coffee, matte tea and soda. These foods will cause Kidney energy deficiency, which in Chinese medicine is responsible for the formation of Yin and Yang energy. [20] As demonstrated in the three case reports, all of them had a deficiency in Yin and Yang Kidney energy, therefore, the removal of these three drinks was important to avoid the worsening of this condition. Patients were also instructed to ingest one liter of water for each $25 \mathrm{~kg}$ of weight, to maintain adequate hydration. [20] The last food group that patients are advised to avoid are: fried foods, chocolate, eggs, coconut, pepper, honey and alcoholic beverages. Because these foods cause the formation of more internal Heat that these patients normally already have, worsening the accumulation of internal Heat responsible for the formation of cancer in the energy level. [20] Auricular acupuncture is a very important asset for TCM. According to TCM, diseases come from imbalances of Qi energy and the goal of auricular acupuncture, therefore, is to restore the needed balance for a healthy body, correcting any distress in the flow of energy by using auricular acupuncture points. The author usually uses auricular acupuncture to regulate the equilibrium between Yin, Yang, Qi and Blood, that usually are the roots of the problem. Usually treating these four energies disharmonies all patients' symptoms and diseases can be treated at the same time. The explanation of each auricular acupuncture's point is well established in the author's article "How Do You Treat Back Pain in Your Practice? Part 2" published on Medical Acupuncture Journal [21].

In Brazil, acupuncture is considered a medical specialty by the Federal Medical Council since 1995. In the article "Anticancer Activities and Mechanisms of Heat-Clearing and Detoxicating Traditional Chinese Herbal Medicine" by Yulin Zhang, Yeer Liang, and Chengwei He, the authors say that according to TCM, pathogenic Heat and Toxins, which can seem to be an inflammation symptom, are actually the causes of cancer and will promote its development. To remove Heat retention, in cancer patients, the author uses the apex-ear bloodletting technique extensively reported in several of her published articles. [22] In the case of cancer patients, as it was said that they have a great energy deficiency, moxibustion is done to replace this energy that patients lack, normally used in points in the stomach to improve appetite (as they usually have a great loss of appetite) and kidney points to improve Yin and Yang energy. The author uses the technique of radiesthesia to measure the amount of energy that each massive organ (Liver, Heart, Spleen, Lungs and Kidney), which is the science of using the vibrational fields 
of the human body to access information about other objects of animate or inanimate nature by establishing resonance with their energy fields. Radiesthesia uses a crystal pendulum to measure the energy of each chakra. Depending on the movement of the crystal, clockwise, or counterclockwise or the crystal's rotation amplitude, it is possible to evaluate the amount of energy that each chakra presents. [14] The chakras correspond to the five elements within the Five Elements Theory, as exposed in small trials in literature. Many ancient medical scholars believe that the five elements theory and the chakras theory can connect. This theory is used extensively by the author in various treatments and scientific publications that can be found in the previously cited articles [14] (Figure 6) (Table $3)$.

Table 3: Medications chosen in the treatment according to the correspondence of chakras and Five Elements.

\begin{tabular}{|l|c|c|c|}
\hline Chakras & Five Elements & Homeopathy Medications & Crystal Based Medications \\
\hline $1^{\circ}$ chakra & Wood/Liver & Phosphorus & Garnet \\
\hline $2^{\circ}$ chakra & Water/Kidney & Natrium muriaticum & Orange Calcite \\
\hline $3^{\circ}$ chakra & Fire/ Heart & Sulphur & Rhodochrosite \\
\hline $4^{\circ}$ chakra & Metal/Lung & Silicea & Emerald \\
\hline 5 hakra & Earth/Spleen & Calcarea carbonica & Blue Quartz \\
\hline
\end{tabular}

In patients diagnosed with cancer, the treatment of this lack of energy may serve as an adjunct to the chemotherapy treatment that the patient is already undergoing - as in the case study patient example - the metastases and the size of the patient's tumor has only been reduced and disappear with the treatment of rebalancing the energies and replenishing of the chakra's energies. In case report three, when only chemotherapy, radiotherapy or surgery was prescribed to the patient, he would always have relapses and the appearance of metastasis after a short period of tumor reduction. [4-7] The author, in her daily clinical practice, in order to improve the vital energy of patients, whenever possible, uses highly diluted medication, leaving the medication in high concentrations only in cases where they are essential for the treatment of the patient. According to Hippocrates, "first do no harm" [19]. The explanation of why the author uses high diluted medication is extensively explained in several works published by the author [4-7] (Figure 7).

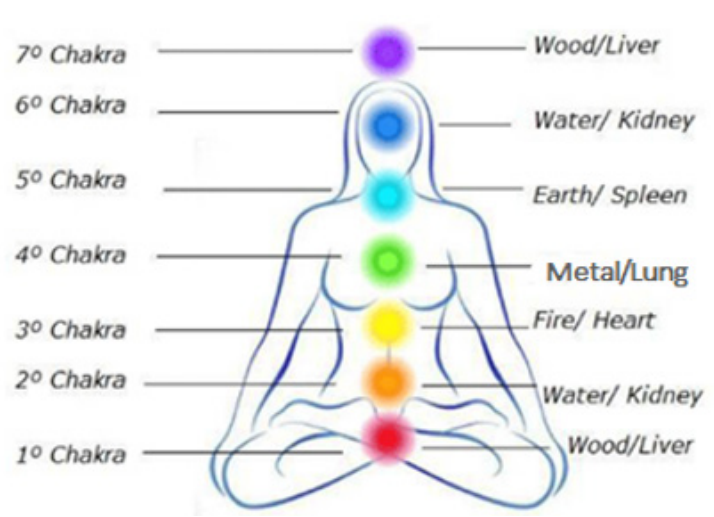

Figure 6: Chakras and their Correspondent Organs According to TCM.

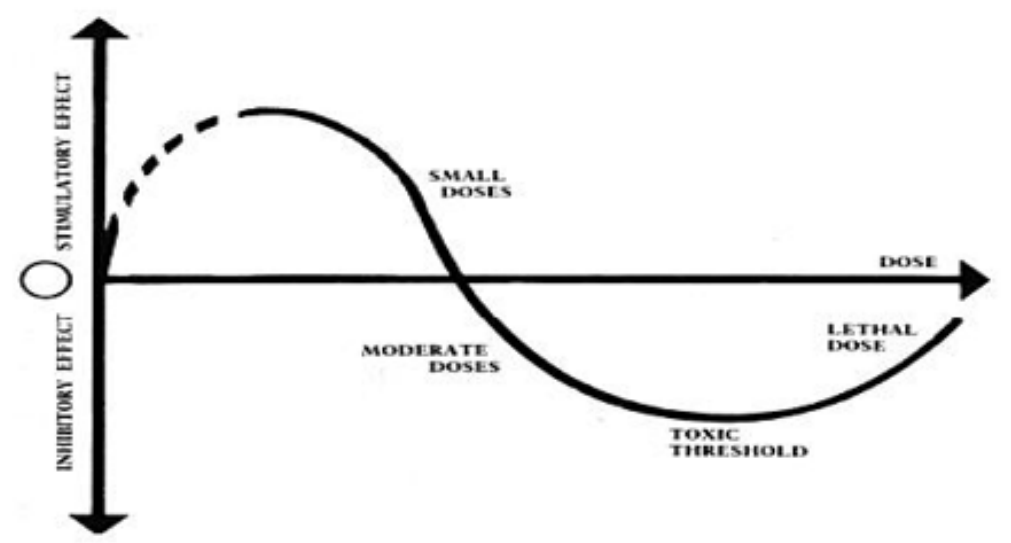

Figure 7: Cycle Explanation of the Arndt-Shultz Law. 
What the author proposes in the treatment of cancer patients is the need to integrating the teachings of TCM with Western medicine; since the treatment of energy imbalance is very important for the recovery of the physical, mental, emotional symptoms of cancer patients. And counterbalancing the reduction in vital energy caused by Western medicine treatment, leading to an improvement in vital energy that is usually very weak in these patients [4-7].

\section{Conclusion}

The conclusion of this study is that it is very important to treat the cancer patient at the energy level. Each patient has their own energy disturbances and the correction of these imbalances is the main point to achieve success in the treatment. As stated by TCM that, cancer is originated from energies deficiencies and Heat retention. In this study, the author demonstrated that all patients in the three clinical cases had in common a profound deficiency in the energy of the chakras, the replenishment of the chakra's energy meridian was important to improve or cure the cancer. Rebalancing the Yin, Yang, Qi and Blood energies and withdrawing the Heat retention besides recharging the chakras' energies, usually very low on these patients. A more comprehensive study should be done involving more patients with different sets of tumors and cancers, to the goal of exploring, improving, and complementing the worldwide treatment of cancer in a holistic way.

\section{Acknowledgment}

None.

\section{Conflict of Interest}

No conflicts of interest.

\section{References}

1. NIH Curriculum Supplement Series (2007) Understanding Cancer National Institutes of Health (US); Biological Sciences Curriculum Study. Bethesda (MD): National Institutes of Health (US).

2. Cooper G M (2000) The Cell: A Molecular Approach. 2nd edition. Sinauer Associates.

3. Liu J, Shuo W, Ying Z, Hui-ting F, Hong-sheng L (2015) Traditional Chinese medicine and cancer: History, present situation, and development.

4. Huang Wei Ling. Why are Diabetic Patients Still Having Hyperglycemia despite Diet Regulation, Anti-glycemic Medication and Insulin? International Journal of Diabetes \& Metabolic Disorders.
5. Huang Wei Ling (2018) Can Recurrent Furunculosis be Treated without the Use of Antibiotics? Acta Scientific Microbiology 1(9): 04-12.

6. Huang Wei Ling (2018) Could Postsurgical Nosocomial Cellulitis be Treated without the Use of Antibiotics? Acta Scientific Microbiology $1(9)$

7. Huang WL (2018) Can Hospital Osteomyelitis Be Treated Without the Use of Antibiotics? Int J Microbiol Infect Dis 2(1):1-6.

8. Weir HK, Thompson TD, Soman A, Møller B, Leadbetter S (2015) The Past, Present, and Future of Cancer Incidence in the United States: 1975 Through 2020.

9. Li X, Yang G, Li X, Zhang Y, Yang J, et al. (2013) Traditional Chinese Medicine in Cancer Care: A Review of Controlled Clinical Studies Published in Chinese.

10. Paddock C (2014) Immune System Kills Spontaneous Blood Cancer Cells Every Day.

11. Naoshing N (1995) The Yellow Emperor's Classic of Medicine: A New Translation of the Neijing. Shambhala Publications.

12. Zampirolli AP (2016) Câncer segundo a MTC. INSTITUTO LONG TAO

13. Yulin Z, Yeer L, Chengwei H (2017) Anticancer Activities And Mechanisms Of Heat-Clearing And Detoxicating Traditional Chinese Herbal Medicine.

14. Bing O, Zhen G (1996) Essentials of Traditional Chinese Medicine. Translated by Lu Yubin. Beijing, China. Ed. Shandong Science and Technology Press.

15. Salman T (2016) Spontaneous tumor regression. Journal of Oncological Science. 2(1): 1-4.

16. Huang Wei Ling (2020) Constitutional Homeopathy of the Five Elements based on Traditional Chinese Medicine. Acta Scientific Medical Sciences.

17. Coimbra O (2017) Semeando a Luz - Cristais de OZ. Editora Chiado.

18. Yin HH, Zhang BN (2012) Teoria Básica da Medicina Tradicional Chinesa. Editora Atheneu.

19. Yapijakis C (2009) Hippocrates of Kos, the father of clinical medicine, and Asclepiades of Bithynia, the father of molecular medicine.

20. Arantes AM (2015) Dietoterapia Chinesa - Nutrição Para Corpo, Mente. Roca; Edição.

21. Asher G, Jonas DE, Coeytaux RR, Reilly AC, Loh YL, et al. (2010) Auriculotherapy for Pain Management: A Systematic Review and MetaAnalysis of Randomized Controlled Trials.

22. Zhang Y, Liang Y, He C (2017) Anticancer Activities and Mechanisms of Heat-Clearing and Detoxicating Traditional Chinese Herbal Medicine. 